low-frequency homology-directed repair. Using inDelphi to evaluate pathogenic, short indels, the team identified approximately 1,600 frameshifts or microduplications that could be reverted to wild-type sequence by template-free Cas 9 editing. Assembling the predicted gRNAs into a second library, they observed $>50 \%$ of correct repair outcomes in 183 and 508 alleles, respectively. Impairment of NHEJ, which biases repair toward microhomology-mediated outcomes, further increased the rate of correction to wild-type sequences, for example, up to $90 \%$ at alleles of $P K D 1, M S H 2$ and $L D L R$.

Finally, the group turned to patient-derived fibroblasts with microduplication alleles in either HPS1, a pathogenic mutation that gives rise to Hermansky-Pudlak syndrome (a disorder clinically characterized by blood clotting deficiencies and albinism), or $A T P 7 A$, which has been linked to copper deficiencies. Using inDelphi-predicted gRNAs, the team achieved an $88 \%$ and $94 \%$ reversion to wild-type sequence, respectively.
Taken together, the authors demonstrate that repair by nonhomologous or microhomologymediated pathways can be computationally predicted and validated in vivo. This predictive power can help target and repair pathogenic mutations caused by indels without requiring a DNA template. However, the authors caution that therapeutic application needs to be considered on a case-by-case basis as not all predicted gRNA and target pairs led to accurate correction of the mutation.

Looking forward, incorporation of large data sets of other repair outcomes, such as long deletions or translocations, will enable inDelphi to make more accurate predictions of repair outcomes at any given target site. With the right training data, inDelphi may learn to predict the outcomes of other designer nucleases, expanding its potential to treat genetic diseases.

Ross Cloney, Senior Editor, Nature Communications

ORIGINAL ARTICLE Shen, M. W., Arbab, M. et al. Predictable and precise template-free CRISPR editing of pathogenic variants. Nature https:// doi.org/10.1038/s41586-018-0686-x (2018) authors speculated that changes in the methylome and transcriptome might be associated with chronic and acute health conditions, respectively.

In support of this hypothesis, the highest percentage of differentially methylated genes (including those associated with glucose-related and diabetes mellitus-related pathways) occurred at 80 and 90 days before glucose elevation, in line with the highest number of DMRs occurring at these times. By contrast, changes in gene expression during periods of viral infection were associated with immune-related pathways. Furthermore, cytosines that were differentially methylated between high and low glucose events were more enriched at promoter regions, TSSs and transcription factor binding sites than cytosines that were differentially methylated between viral infection and non-viral infection states; these data suggest that changes in glucose level influence gene expression through promoter DNA methylation more than viral infections do.

Finally, as the authors had previously identified which alleles were specific to either maternal or paternal chromosomes, they could assess allele-specific methylation (ASM) patterns over the time course. ASM patterns were stable over the 26 time points assessed; this enabled the authors to combine the WGBS reads from all samples and characterize ASM patterns at an unprecedented depth. This characterization indicated a higher density of allelic DMRs (aDMRs) on chromosomes 4, 17 and 19 and showed that the number of DMRs correlated with the number of genes in each chromosome; thus, aDMRs might help to regulate gene expression.

This study indicates that, at least for this individual, the DNA methylome and transcriptome are associated with different health-related events and that personalized DNA methylomics might predict disease.

Katharine H. Wrighton

ORIGINAL ARTICLE Chen, R. et al. Longitudinal personal DNA methylome dynamics in a human with a chronic condition. Nat. Med. https://doi. with a chronic condition. Nat. Med. https:///di
org/10.1038/s41591-018-0237-x (2018) FURTHER READING Karczewski, K. J. \& Snyder, M. P. Integrative omics for health and disease. Nat. Rev. Genet. 19, 299-310 (2018)

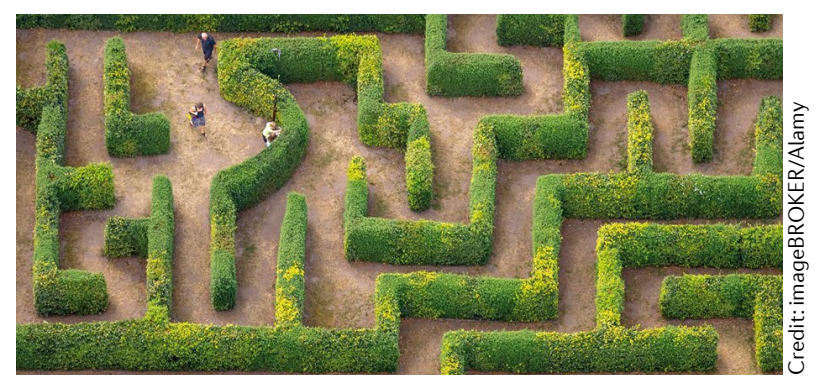

CANCER GENOMICS

\section{Cancer chromatin accessed}

The Cancer Genome Atlas (TCGA) has been instrumental in studying genomic and epigenomic aberrations in a multitude of different human cancers. Corces, Granja et al. now report a rich data resource of accessible chromatin regions for 23 cancer types that provides insight into the landscape of active regulatory DNA elements and transcriptional regulation in human tumours.

A total of 410 tumour samples were used as input for assay for transposase-accessible chromatin using sequencing (ATAC-seq), which identified more than 500,000 pan-cancer peaks of chromatin accessibility. These peaks overlapped with promoter and enhancer regions defined by chromatin immunoprecipitation followed by sequencing (ChIP-seq) in the ChromHHM database, as expected. Distal elements showed stronger cancer type specificity, whereas proximal, promoter regulatory elements showed similar patterns across cancers.

Based on the genome-wide ATAC-seq patterns, cancer types were clustered into different groups, and cluster-specific peak sets were observed to be enriched for relevant transcription factor (TF) motifs as well as genetic variants identified in genome-wide association studies for these cancers. New cancer subtypes could be defined on the basis of these chromatin accessibility patterns.

Correlating the ATAC-seq peaks with matched RNA sequencing data, the authors were able to predict peak-to-gene links, both across all cancers and specifically for breast cancer. To validate a subset of these links, a CRISPR interference (CRISPRi) approach was used to introduce heterochromatin signatures at select distal peaks.

Peaks targeted in this way were associated with a decrease in expression of the linked gene, located kilobases or megabases away. These assays also emphasized the cell type and cancer type specificity of the peak-gene relationships, for example for BCL2 in luminal-like but not basal-like breast cancer.

Complementary to this unique resource, future studies will be crucial to delineate the contribution of cellular composition of the ATAC-seq signal, to include (matched) healthy tissues and to add additional genomic data such as 3D chromosome conformation to enable researchers to home in on the causative genomic regions in a wide range of human cancers at the full level of complexity of the tumour tissue.

Michelle Trenkmann, Associate Editor, Nature Communications

ORIGINAL ARTICLE Corces, M. R., Granja, J. M. et al. The chromatin accessibility landscape of primary human cancers. Science 362, eaav1898 (2018) 